\title{
Der Rollstuhl auf der Treppe
}

\section{Adrian Ritter}

Freier Journalist

\author{
Mit dem "Cybathlon» bot die ETH Zürich Anfang Oktober eine Leistungsshow \\ der Rehabilitationstechnologie. An einem Podium wurde parallel dazu diskutiert, \\ inwiefern Hightech der Schlüssel zur Gleichstellung von Menschen mit Behinde- \\ rung sein kann.
}

Wer kann mit einer Armprothese am schnellsten den Tisch decken oder die Jacke zuknöpfen? Wer schafft es, mit einem neuartigen Rollstuhl eine Treppe runterzufahren? Beim «Cybathlon» der ETH Zürich traten am 8. Oktober Menschen mit einer körperlichen Behinderung in sechs Disziplinen zum Wettkampf an. Gefragt waren allerdings nicht sportliche Höchstleistungen im Sinne der Paralympischen Spiele. Vielmehr stand die Frage im Zentrum, inwiefern Rehabilitationstechnik geeignet ist, Menschen mit körperlichen Behinderungen im Alltag eine Hilfe zu sein. Die rund 70 Teams aus aller Welt testeten dabei sowohl Prototypen aus Forschungslabors wie auch Produkte, die bereits auf dem Markt erhältlich sind.

\section{Selbstbewusst mit «Makel»}

Aber inwiefern ist Hightech wirklich der Schlüssel zur Gleichstellung von Menschen mit einer körperlichen Behinderung? Dieser Frage widmete sich im Vorfeld des «Cybathlon» ein Podiumsgespräch an der ETH Zürich. Initiiert hatte es Brian McGowan, Präsident von Sensability - einem Verein, der Beratung zu den Themen Gleichstellung und Behinderung anbietet.

\section{Nicht nur treppengängige Hightech-Rollstühle} sind gefragt, sondern auch weniger Treppen.

Er sei aufgrund seiner Muskelerkrankung seit der Kindheit froh um den elektrischen Rollstuhl, sagte McGowan in seinem Inputreferat. Nur die Einschränkungen eines Menschen in den Vordergrund zu stellen und nach technischen Lösungen dafür zu suchen, sei aber gefährlich. «Das wäre eine Rückkehr zu einem Modell der Behinderung, wie es bis in die 1980er Jahre vorherrschend war», so McGowan. Ein zeitgemässeres Verständnis - wie es auch in der UN-Behindertenrechtskonvention zu finden sei - anerkenne, dass Be-

\section{Bericht zur UN-Behindertenkonvention}

Im Juni 2016 hat der Bundesrat den ersten Bericht der Schweiz an die UNO zur Umsetzung des Übereinkommens über die Rechte von Menschen mit Behinderungen («Behindertenkonvention») veröffentlicht. Der Bericht zeichnet ein positives Bild: Das Behindertengleichstellungsgesetz, verschiedene Revisionen der Invalidenversicherung und das neue Erwachsenenschutzrecht hätten wesentliche Verbesserungen für Menschen mit Behinderungen gebracht. Die Selbstbestimmung und die Teilhabe von Menschen mit Behinderungen würden zudem gefördert durch die sozialstaatliche Eingliederungspolitik, etwa im Rahmen der Invalidenversicherung oder durch die verbesserte Zugänglichkeit zu Bauten oder zum öffentlichen Verkehr.

Der Bericht zeigt aber auch Handlungsbedarf auf. Das Eidgenössische Departement des Innern hat deshalb die Aufgabe, bis Ende 2016 einen Bericht zur Behindertenpolitik vorzulegen. Dieser soll Vorschläge enthalten, wie die bestehenden behindertenpolitischen Massnahmen von Bund und Kantonen besser aufeinander abgestimmt werden können und wie in Bereichen wie Bildung oder Arbeit die Gleichstellung von Menschen mit Behinderungen weiter gefördert werden kann.

hinderung ein Wechselspiel zwischen individueller Beeinträchtigung und Hindernissen in der Umwelt sei. «Der technologische Fortschritt sollte auch Hindernisse abbauen helfen", so McGowan. Nicht nur treppengängige Hightech-Rollstühle sind gefragt, sondern auch weniger Treppen. «Das ist nicht nur im Interesse von Menschen mit einer Behinderung, sondern macht auch älteren Menschen und Eltern mit Kinderwagen das Leben leichter», so McGowan.

Technik solle nicht mit immer kleineren Hilfsmitteln und unauffälligeren Prothesen helfen, den körperlichen «Makel» zu verstecken, forderte McGowan. Was normal ist, definiere ohnehin die Gesellschaft: «Selbstbestimmt leben können in einer möglichst hindernisfreien Umwelt heisst auch, sich mit einem 'Makel' in der Öffentlichkeit zu bewegen. Nur so werden auch Hindernisse in den Köpfen abgebaut, also Vorurteile.» 


\section{Fluch der Verbesserung}

Am anschliessenden Podiumsgespräch wurden weitere Aspekte des Verhältnisses von Technologie, Behinderung und Gesellschaft diskutiert. Mit Robert Riener, als Professor für Sensomotorische Systeme an der ETH Zürich und an der Universitätsklinik Balgrist tätig, war

Technik solle nicht mit immer kleineren Hilfsmitteln und unauffälligeren

Prothesen helfen, den körperlichen «Makel» zu verstecken, forderte McGowan.

auch der Initiant des "Cybathlon» mit dabei. Gemäss Riener will die Veranstaltung gerade auch zeigen, dass die Rehabilitationstechnologie noch lange nicht so alltagstauglich ist, wie man sich das wünscht: «Der Bedarf an Entwicklung ist noch gross.»

Die stete Verbesserung des menschlichen Körpers durch Technologie könne auch ein Fluch sein, gab Karin Harrasser, Kultur- und Medienwissenschaftlerin an der Kunstuniversität Linz, zu bedenken. Dies etwa darum, weil schnell ein gesellschaftlicher Druck entstehen könne auf Menschen mit Behinderung, den eigenen Körper technologisch zu optimieren.

Gemäss Joe Manser, Leiter der Schweizerischen Fachstelle für behindertengerechtes Bauen, besteht bereits eine Tendenz in diese Richtung. Technologie sei einer-

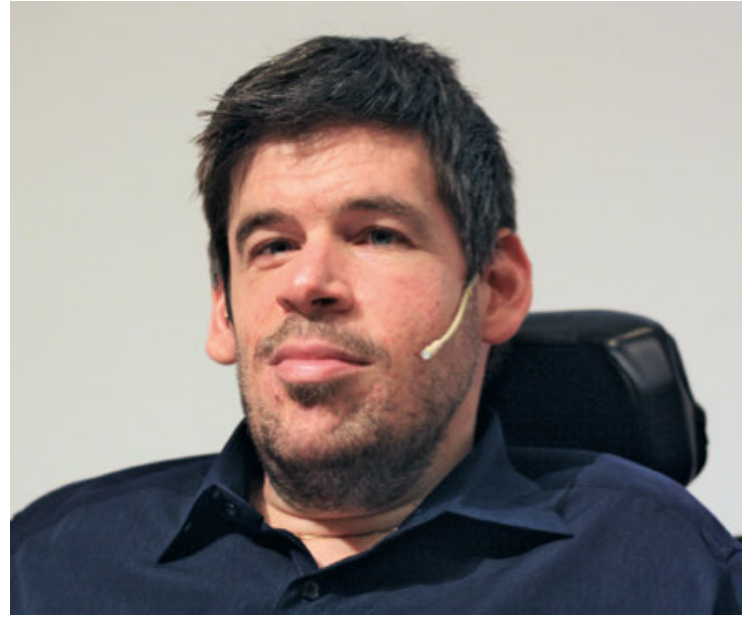

Plädiert für ein Verständnis von Behinderung, welches das Wechselspiel zwischen individueller Beeinträchtigung und Hindernissen in der Umwelt berücksichtigt: Brian McGowan, Präsident des Vereins Sensability.

(Foto: Adrian Ritter)

seits tatsächlich ein Mittel zur Gleichstellung. So seien früher Telefonkabinen nicht rollstuhlgängig gewesen. Das Problem habe sich mit dem Aufkommen der Mobiltelefone erledigt - diese erlauben Gehörlosen erst noch die schriftliche Kommunikation per SMS. Ein Fortschritt also. Andererseits sieht Manser die Gefahr, dass vor lauter Technik für das Individuum tatsächlich auf Umweltveränderungen verzichtet wird.

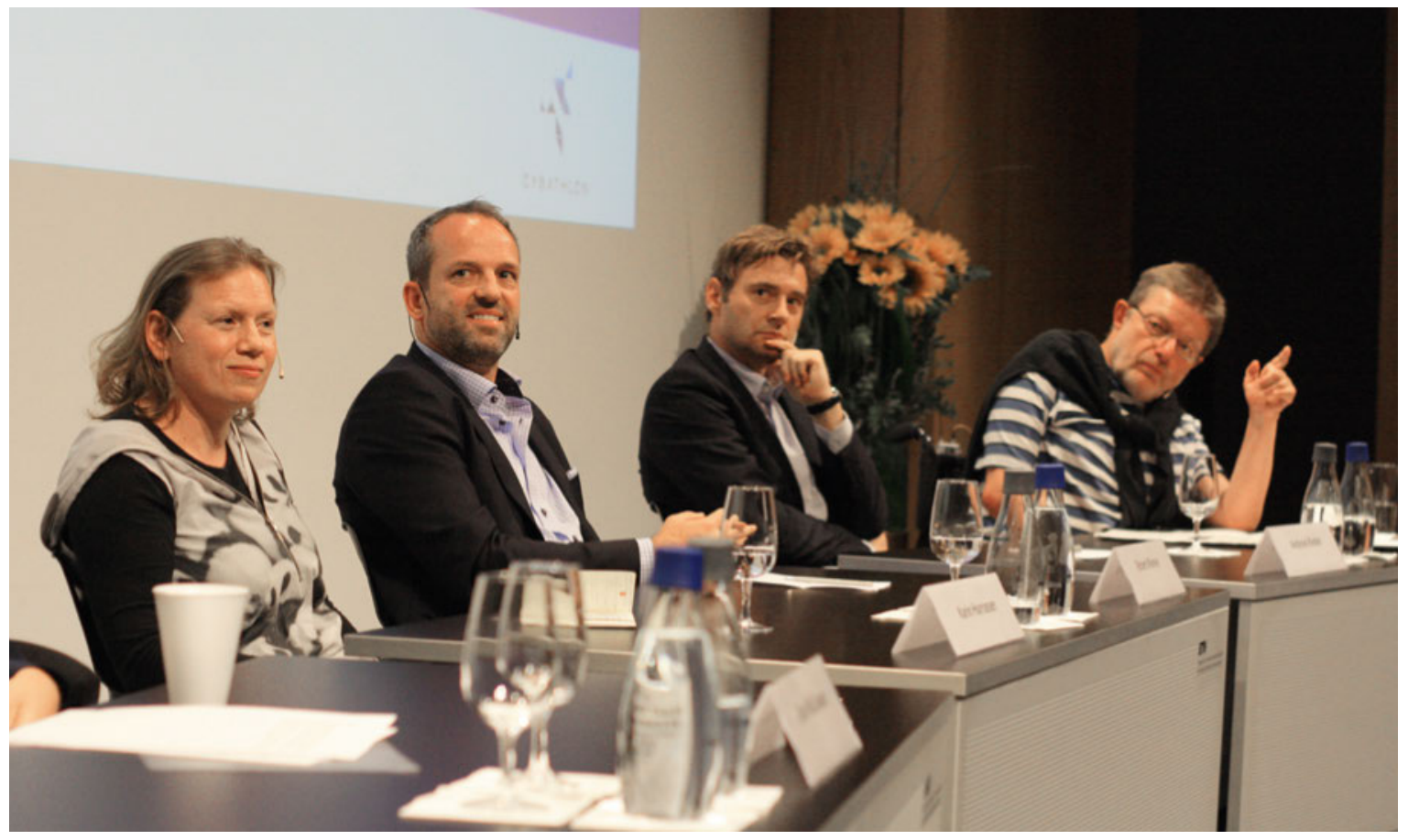

Die weiteren Teilnehmenden des Podiumsgesprächs zu Technologie und Gleichstellung (von links): Karin Harrasser (Kunstuniversität Linz), Robert Riener (ETH Zürich), Andreas Rieder (Eidgenössisches Büro für die Gleichstellung von Menschen mit Behinderung) und Joe Manser (Schweizerische Fachstelle für behindertengerechtes Bauen). 


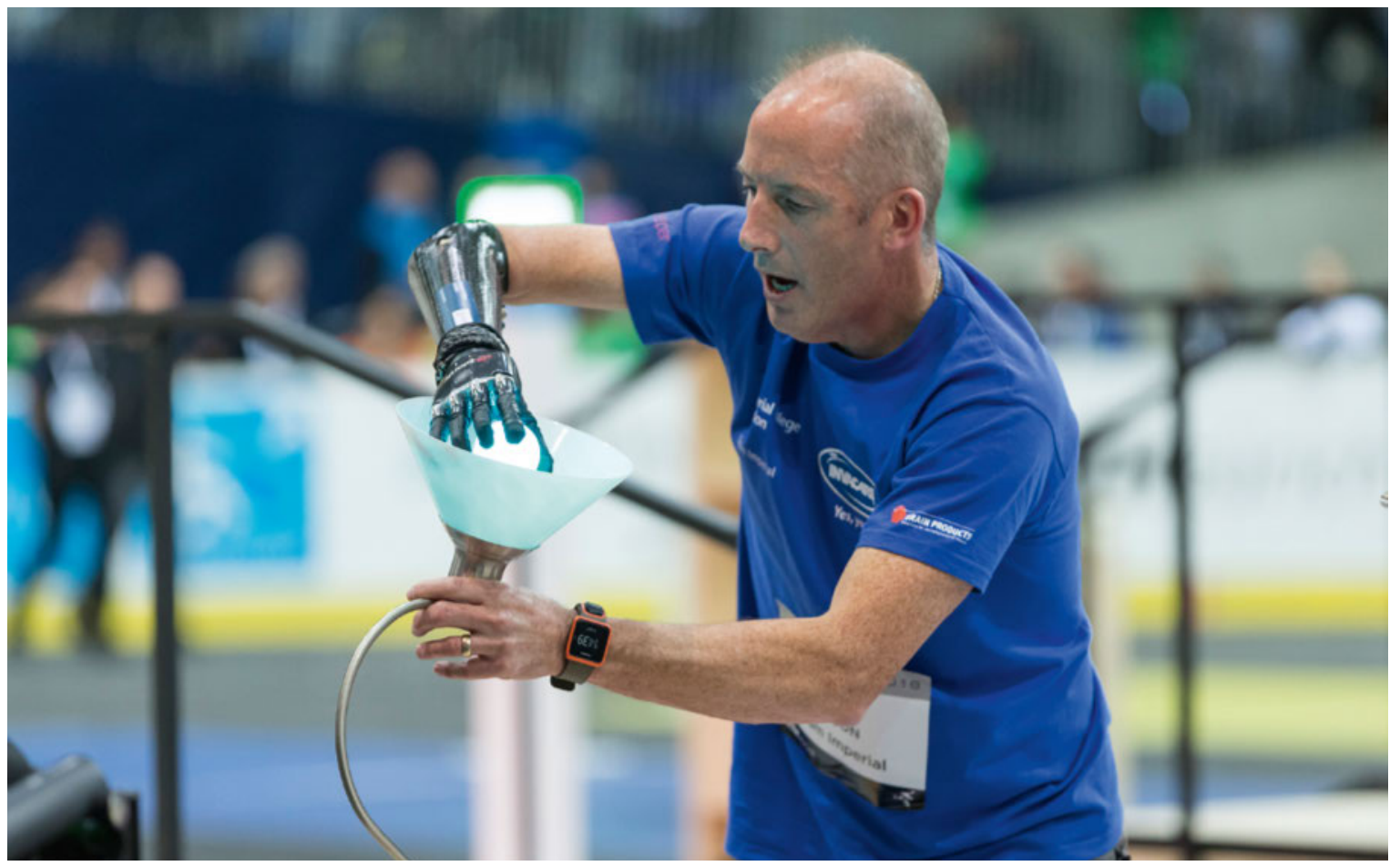

«Cybathlon»: Bei der Veranstaltung der ETH Zürich ging es am 8. Oktober 2016 darum, die Alltagstauglichkeit von Rehabilitationstechnologien zu testen.

Aber auch technologische Rehabilitation ist für Menschen mit Behinderung nicht beliebig verfügbar. Gemäss Joe Manser ist die Invalidenversicherung nicht mehr so grosszügig wie früher, was die Kostenübernahme anbelangt. Neben der Gefahr, zur Nutzung von Technologie gezwungen zu werden, bestehe somit umgekehrt auch die Gefahr, dass sich Menschen mit einer Behinderung innovative technische Hilfsmittel gar nicht leisten können. CVP-Nationalrat Christian Lohr als Gast im Publikum forderte denn auch, Menschen mit Beeinträchtigungen nicht primär als Kostenfaktor zu sehen.

Andreas Rieder brachte als Leiter des Eidgenössischen Büros für die Gleichstellung von Menschen mit Behinderung einen weiteren Aspekt in die Diskussion ein. Bei der Frage, was Technologie zur Gleichstellung beitragen könne, gehe es zumeist um Menschen mit körperlichen Behinderungen. Daneben dürften aber die Bedürfnisse von Menschen mit psychischen und geistigen Behinderungen nicht vergessen werden.
Die Teilnehmenden des Podiumsgesprächs waren sich einig, dass es sinnvoll ist, offen zu sein für technologische Entwicklungen. Menschen mit einer Behinde-

\section{Gemäss Joe Manser ist die Invaliden-}

versicherung nicht mehr so grosszügig wie

früher, was die Kostenübernahme anbelangt.

rung müssten aber selbstbestimmt entscheiden können, welche Technologien sie nutzen wollen. Zentral sei zudem, Betroffene noch viel stärker in die Entwicklung von technologischen Innovationen einzubeziehen.

\section{Video des Podiumsgesprächs}

Die Videoaufzeichnung des Podiumsgesprächs ist auf der Website des "Cybathlon» verfügbar: http://www.cybathlon.ethz.ch 